\title{
CULTURA CÍVICA UNIVERSITARIA DE LA SALLE PACHUCA DESDE LA PERCEPCIÓN DE LOS ESTUDIANTES DE LICENCIATURA
}

\section{CIVIC CULTURE IN THE LA SALLE UNIVERSITY OF PACHUCA THE PERCEPTION OF UNDERGRADUATE STUDENTS}

\author{
Humberto Mejía Zarazúa Maestro en \\ Ciencias de la Educación y colaborador de la Dirección de Posgrado e \\ Investigación de la Universidad La Salle Pachuca, \\ humberto.mejia@lasallep.edu.mx
}

\section{Resumen}

En el presente reporte de investigación se propone el concepto Cultura Cívica Universitaria y su operacionalización a través de un ejercicio de tipo ideal, diseño de instrumento de investigación y una encuesta realizada en la Universidad La Salle Pachuca en mayo de 2010. Se consideraron tres ámbitos: el curricular, el institucional y el extraescolar. Se exponen los resultados por ámbito y por licenciatura, y se concluye que mediante esta propuesta es posible diagnosticar el estado de la formación ciudadana en las Instituciones de Educación Superior.

Palabras clave: Cultura Cívica Universitaria, La Salle Pachuca, curricular, Institucional, extraescolar.

\section{Abstract}

In this research we propose the concept of University Civic Culture and the way it is measured through an ideal type of exercise, the design of the research's instrument and a survey conducted at La Salle Pachuca University in may 2010. There were three areas considered: curricular, institutional and extra-curricular. Results were exposed by field and degree and it was concluded that it is possible to diagnose the state of civic education in higher education institutions through this proposal.

Key words: University Civic Culture, La Salle Pachuca University, curricular, institutional, extra-curricular.

\section{Antecedentes}

Precedente a este reporte de investigación, en el número 08 de Xihmai se publicó el referente a la "cultura política de estudiantes de licenciatura en la Universidad La Salle Pachuca" (Mejía, 2009), en él se señalan algunos referentes sobre la dimensión política en la Educación Superior para enfatizar que las universidades lasallistas no son ajenas a ella. Los resultados señalaron que, aunque el alumnado tiene un claro espíritu democrático en el que prevalece una posición principalmente culturalista e institucional como vías para mejorar nuestra democracia, ésta se valora deficientemente al igual que el Estado de derecho. Su principal preocupación sociopolítica es la corrupción y la pobreza, existe también un descrédito acerca de la clase política mexicana. Por otro lado, es un estudiantado con visión multicultural, al menos en lo que se refiere al respeto hacia el otro 
respecto a condición social, cultural, religiosa o de preferencia sexual; asimismo la familia y/o la localidad de residencia son las fuentes principales de formación de valores, por encima de la Universidad. En esta aportación, se propone el concepto Cultura Cívica Universitaria y se presentan los resultados que describen dicha cultura en el estudiantado de licenciatura.

Para plantear el problema de investigación se parte de la discusión teórica alrededor de la formación ciudadana en la educación superior (trabajo previo con abundantes referencias), a fin de proponer como constructo el concepto de Cultura Cívica Universitaria (CCU), en este caso, mediante un ejercicio de tipo ideal (Weber, 1984), de las actitudes, conocimientos y valoraciones (Almond y Verba, 1992) que el estudiante universitario poseería, circunscritos en tres ámbitos: el curricular, el institucional y el extraescolar, de manera que en términos de preguntas de investigación, se plantearon las siguientes:

¿Qué elementos pueden configurar un constructo relativo a la Cultura Cívica Universitaria?

¿Cuál es la valoración de los estudiantes de licenciatura de la Universidad La Salle Pachuca en cuanto a la aportación de su programa académico a su CCU?

¿Cuál es la valoración de los estudiantes de licenciatura de la Universidad La Salle Pachuca en cuanto a la aportación de la institución a su CCU?

¿Cuál es la valoración de los estudiantes de licenciatura de la Universidad La Salle Pachuca en cuanto a la aportación de las relaciones sociales extraescolares a su CCU?

El principio orientador es desarrollar este constructo mediante tres ámbitos estructurales: las especificidades curriculares del programa académico, el ámbito institucional y el ámbito extraescolar. Los dos primeros derivan de la institución universitaria propiamente dicha, el tercero básicamente se refiere a las redes sociales que el alumnado establece fuera de la universidad. Estos tres ámbitos se pueden configurar mediante el planteamiento de Almond y Verba (1992) relativo a la cultura política, pero circunscritos a la educación universitaria y en relación con la formación ciudadana. En este sentido se han incorporado en la configuración de la $\mathrm{CCU}$, algunas valoraciones, actitudes, habilidades y conocimientos (lo que algunos consideran competencias).

El ejercicio de operacionalización del concepto propuesto (CCU) se aplicó en la Universidad La Salle de Pachuca en mayo de 2010, mediante una encuesta que cubrió el 84 por ciento de la matrícula total. Es decir, de una población estudiantil de 574 alumnos, se encuestaron a 485 estudiantes de doce licenciaturas integradas en la Facultad de Ciencias Humanas, la Facultad de Ciencias Administrativas, la Escuela de Ingeniería y las licenciaturas de Derecho y Enfermería.

\section{Método}

Como parte del método científico de la sociología comprensiva, Weber (1984) establece la construcción de tipos (tipo ideal) a través de un ejercicio racional con arreglo a fines y/o con arreglo a valores, es decir, es un recurso metódico que no necesariamente tiene que ver con la realidad. M. Laura Velasco (2001, p. 300), explica que el tipo ideal Weberiano es un procedimiento metodológico que concibe un orden social "construido" 
conceptualmente, resultado de aislar ciertos aspectos de un fenómeno. El investigador, entonces, realiza una construcción hipotética como instrumento metodológico para la comparación empírica y captar determinadas especificidades que se alejan de ese modelo en un referente de contrastación. El concepto Cultura Cívica Universitaria se consideró como aquel conjunto de conocimientos, habilidades, actitudes, valoraciones y acciones planificadas que poseen los estudiantes universitarios, adquiridas en los ámbitos curricular (estudio de una profesión), institucional (universidad) y extraescolar (proceso de socialización), que interactúan y están relacionadas con su proceder ciudadano. Bajo este modelo, la formación ciudadana requiere de la construcción de un núcleo estable de índole cognitivo y cultural, "Cuáles deben ser los contenidos de este núcleo "duro» del desarrollo cognitivo y cultural y cómo se deciden, constituyen motivos de debate muy importante" (Tedesco, 2007, p. 158).

Las características del tipo ideal de CCU que debe poseer el estudiante son variadas, uno de los aspectos del sujeto se refiere a que está informado, lo cual implica que puede localizar, discriminar, valorar y utilizar diversas fuentes de información; sabe relacionarla y establecer hipótesis, asimismo tiene la capacidad de reconsiderar o modificar puntos de vista de acuerdo con la información que acumule; conoce los derechos y obligaciones ciudadanas, así como los objetivos y el quehacer institucional. Conoce el contexto sociopolítico en el que está inmerso y sabe de los grupos y movimientos sociales, así como de las demandas de las comunidades locales y regionales. Es un sujeto participativo pues tiene la capacidad de asociación para generar capital social y la capacidad de expresar demandas. Fomenta una participación adecuada al contexto y a las características individuales de las personas. Ejerce sus derechos ciudadanos y genera proyectos comunes de desarrollo social, además cuenta con la capacidad de convivir con grupos humanos vulnerables. Puede ejercer un liderazgo o participar en el desarrollo de proyectos con la comunidad de pertenencia con base en la construcción de significados de bienestar colectivo. Genera y desarrolla espacios públicos y se inserta en redes sociales con objetivos públicos y personales.

Es también un sujeto crítico pues desarrolla su facultad deliberativa y procesos de observación y autocrítica. Puede debatir acerca del mensaje textual y cifrado de los medios de comunicación. Es autónomo pues toma decisiones reflexionadas tanto en el ámbito colectivo como en el individual, para éste genera proyecto de vida y autorrealización. Tiene la capacidad de independencia de juicio y participa en procesos de deliberación y diálogo. Se considera que es un sujeto culto que conserva, difunde, participa y disfruta de la creación y/o apreciación cultural, tiene la capacidad de tener una visión holística de los fenómenos sociales y respeta y comprende diversas cosmovisiones. Es capaz de disfrutar la lectura literaria y valora la cultura popular. Asimismo reflexiona acerca de la relación entre lo local y lo global, lo comunitario y lo universal.

Por supuesto, es un sujeto respetuoso y tolerante que reconoce las diferencias culturales y de identidad. Tiene la capacidad de tomar en cuenta las distintas racionalidades e inteligencias; rechaza la discriminación por clase social, género, raza, preferencia sexual, discapacidad o cultura. Respeta los derechos humanos de los grupos culturalmente diferentes y posee la capacidad de vivir 
experiencias de socialización con grupos e individuos con diferente cosmovisión. Asimismo asume una identidad; aunque tiene sentido crítico de la pertenencia a un Estado-nación y a determinada comunidad; es capaz de identificarse como ciudadano del mundo, así como de desarrollar su propia identidad. Otorga sentido a los actos cotidianos, realiza actos de solidaridad y dota al aprendizaje de sentido de vida.

Es un sujeto comunicativo que ejerce la libertad de pensamiento y la capacidad de expresarlo, posee una alta comprensión lectora y un alto nivel de expresión escrita, por lo que puede plantear con claridad ideas, opiniones y demandas, con un alto nivel argumentativo. Por ello es capaz de participar en un debate político, tener un pensamiento plural y escuchar diferentes ideas y puntos de vista. Posee sentido de justicia social, apoya la generación de un proyecto nacional, plantea problemas socialmente pertinentes, genera comportamientos con sentido democrático y es capaz de vincularse con la sociedad civil. Considera el conocimiento más como un medio de desarrollo humano que como una mercancía, posee conciencia ética y social, maneja las nuevas tecnologías de la información y comunicación y es capaz de participar en convocatorias locales, regionales, nacionales e internacionales para manifestar apoyo u oposición a determinadas decisiones políticas económicas y sociales.

Finalmente es competente para el ámbito laboral ya que tiene capacidad de emprender proyectos personales o colectivos ligados a ejercicio profesional y que generen ingresos económicos. Tiene la capacidad para la flexibilidad laboral, sabe enfrentar la incertidumbre y construye conceptos complejos que integran lo analítico y lo holístico. Desarrolla habilidades que resuelvan problemas en su ámbito profesional, participa en redes, tiene sentido del bien común y del desarrollo compartido y posee un sentido de la ética tanto en su pensamiento como en sus actitudes. Tiene capacidad de llevar a cabo hábitos para una vida saludable.

Con base en este ejercicio de tipo ideal se determinaron las variables a considerar en el instrumento de investigación. La principal fuente de información para su diseño fue "Construcción de una escala de actitudes sobre ética profesional de Ana Hirsh" (2005), en la cual uno de sus fundamentos epistemológicos se basa en la capacidad del ser humano, en este caso del alumnado universitario de licenciatura de La Salle Pachuca, de hacer juicios y evaluaciones, considerando competencias cognitivas, sociales, éticas y afectivoemocionales. La valoración de procesos en este caso se operacionaliza mediante la calificación del alumno en una escala del uno al cinco, semejante a una escala Likert donde se fijan dos extremos (mínima y máxima valoración, con un punto medio). La representación de la escala se ha dividido en tres niveles: bajo, medio y alto, quedando de la siguiente manera:

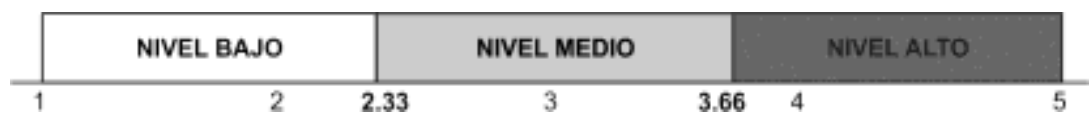

Al dividir en tres niveles y considerando que las ponderaciones van de 1 a 5 , se consideraría bajo el nivel si resulta menor o igual a 2.33, un nivel medio si van de 2.34 a 3.66 y un alto nivel si van de 3.67 a 5. 
El análisis estadístico se realizó con base en el software SPSS. Para el análisis de fiabilidad se utilizó el alfa de cronbach, y para determinar diferencias significativas se utilizó la prueba $T$, tanto para muestras independientes como para muestras relacionadas

\section{Cultura Cívica Universitaria de estudiantes de licenciatura de la Universidad La Salle Pachuca}

Respecto al ejercicio de constructo que se propone y utilizando la media aritmética global, la CCU en los estudiantes de la Universidad La Salle Pachuca, dentro de la escala propuesta es de 3.9 , que corresponde a un nivel alto, aunque cerca del límite con el nivel medio:

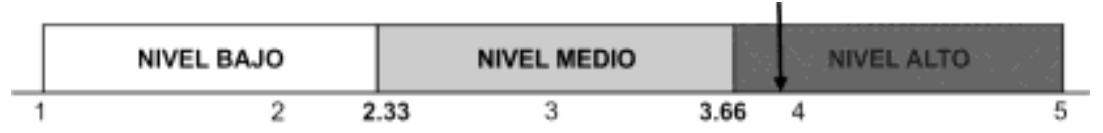

Cabe señalar que las medias aritméticas de cada ámbito, considerando a todas las licenciaturas fue 4.0 para el ámbito curricular, 3.8 para el institucional y 3.9 para el extraescolar. En el siguiente cuadro se anotan los promedios por cada una de las licenciaturas en orden descendente. Como se aprecia los extremos son Enfermería y Tecnología de Información para los Negocios, sin embargo, se considera necesario aclarar que en el caso de Enfermería, los encuestados fueron 17 estudiantes de segundo semestre y en el segundo caso sólo dos estudiantes de cuarto semestre (en ambos casos era la matrícula total). En lo sucesivo habrá que considerar esta situación. Cabe señalar, que excepto la segunda licenciatura mencionada, todas se ubican en un nivel alto, aunque las que integran la Escuela de Ciencias Administrativas y la Escuela de arquitectura y Diseño Gráfico, cerca del límite con el nivel medio.

\begin{tabular}{|l|c|c|c|c|}
\hline \multicolumn{1}{|c|}{ LICENCIATURA } & $\begin{array}{c}\text { AMBITO } \\
\text { CURRICULA }\end{array}$ & $\begin{array}{c}\text { AMBITO } \\
\text { INSTITUCIONA }\end{array}$ & $\begin{array}{c}\text { AMBITO } \\
\text { EXTRAESCOL }\end{array}$ & \\
\hline Enfermería & 4.2 & 4.5 & 4.2 & 4.2 \\
\hline Ing. Cibernética & 4.1 & 4.2 & 3.8 & 4.0 \\
\hline C. de la & 4.2 & 3.9 & 3.9 & 3.9 \\
\hline Derecho & 4.1 & 3.7 & 4.1 & 3.9 \\
\hline Ing. Mecatrónica & 4.0 & 4.0 & 3.9 & 3.9 \\
\hline C. de la Educación & 4.1 & 3.6 & 4.0 & 3.9 \\
\hline Psicología & 4.0 & 3.7 & 3.9 & 3.8 \\
\hline Arquitectura & 3.9 & 3.8 & 3.9 & 3.8 \\
\hline Administración & 3.9 & 3.7 & 3.9 & 3.8 \\
\hline Diseño Gráfico & 4.0 & 3.8 & 3.6 & 3.7 \\
\hline Contaduría & 3.8 & 3.7 & 3.9 & 3.7 \\
\hline Tec. de Inf. para & 2.9 & 3.1 & 4.4 & 3.4 \\
\hline los & & & & 3 \\
\hline
\end{tabular}

Fuente: Encuesta sobre CCU en estudiantes de licenciatura de La Salle Pachuca (mayo de 2010) 


\section{Ámbito curricular}

Las licenciaturas que más valoraron la aportación del ámbito curricular (sus estudios de licenciatura) para su CCU fueron Enfermería (4.2), Ciencias de la Comunicación (4.2), Ingeniería Cibernética (4.1), Derecho (4.1) y Ciencias de la Educación (4.1). Las licenciaturas que más bajo valoraron este aspecto fueron Tecnología de Información para los Negocios (2.9), Contaduría (3.8), Administración (3.9) y Arquitectura (3.9). Aun cuando la valoración más baja es en la licenciatura que cuenta con sólo dos alumnos, llama la atención que las que componen la Facultad de Ciencias Administrativas son las que más apariciones tienen en el nivel medio, Por ejemplo en la variable localizar y valorar la utilidad y calidad de diversas fuentes de información, las tres licenciaturas de la mencionada facultad son las únicas de nivel medio, con una diferencia significativa con Enfermería, Ciencias de la Comunicación y Diseño Gráfico.

Respecto a la variable relacionar información y establecer hipótesis las licenciaturas de la Escuela de Ingeniería (junto con Ciencias de la Educación) son las que valoran más alto la contribución de su programa académico, con una diferencia significativa con las que integran la Facultad de Ciencias Administrativas y Arquitectura, las cuales se ubican en el nivel medio. En la variable reflexionar acerca del mensaje textual y la intención de los mensajes de los medios de comunicación, se considera que se evidencia la influencia curricular al tener la valoración más alta las licenciaturas de Ciencias de la Comunicación y Diseño Gráfico, ya que involucran contenidos que precisamente corresponden a emisión de mensajes. Asimismo esta influencia es clara para la variable resolver problemas en diferentes condiciones y contextos, puesto que son los programas de la Escuela de Ingeniería las más altas en su valoración, debido precisamente al continuo contacto con problemas prácticos y teóricos.

Algo similar sucede con la variable obtener una alta comprensión lectora y nivel de expresión escrita, donde las licenciaturas de Ciencias de la Comunicación y Derecho tienen las más altas puntuaciones, con diferencia significativa con las licenciaturas de la Facultad de Ciencias Administrativas, Diseño Gráfico, Arquitectura e Ingeniería Mecatrónica, que además se ubican en un nivel medio. En el mismo tenor, la variable considerar el conocimiento como un medio de desarrollo humano más que como una mercancía, cuenta con mayor valoración en Ciencias de la Comunicación y Ciencias de la Educación, pertenecientes precisamente a la Facultad de Ciencias Humanas. En la variable respeto por la legalidad, la más alta valoración se obtiene en Enfermería y Derecho, se considera digno de mención este dato por la relación entre la variable y la orientación del programa académico en el caso de Derecho; hay diferencia significativa con Arquitectura y Tecnología de Información para los negocios (TIN), aunque ésta es la única que se valora en un nivel medio. En este sentido lo mismo sucede con la variable Tomar decisiones reflexionadas y tener independencia de juicio, en donde si bien las demás licenciaturas se ubican en el nivel alto, la prueba $\mathrm{T}$ determinó diferencia significativa entre Administración e Ingeniería Mecatrónica, que fue el programa que más valoró esta aportación.

Respecto a generar un proyecto de vida y de autorrealización, así como dotar al aprendizaje de un sentido de vida, todas las licenciaturas (excepto, TIN) la 
ubican en un nivel alto, aunque con una diferencia significativa entre Ingeniería Cibernética, y Enfermería y Ciencias de la Educación que fueron las mayor valoradas, situación coherente con el contenido de la variable y los valores de vocación y servicio de los profesionales de la salud y la educación, procesos que a menudo involucran a personas más convencidas que bien remuneradas.

En relación con el ámbito curricular, por último, la variable conocer derechos y obligaciones ciudadanas, refuerza la influencia curricular, pues es la Licenciatura en Derecho la que más la valora, seguida de Ciencias de la Educación y Administración, estos tres programas tienen diferencia significativa en su valoración con Ciencias de la Comunicación, las dos ingenierías, Diseño Gráfico y TIN, que además ubican la variable en un nivel medio. Llama la atención que dos programas de la Facultad de Ciencias Humanas tengan diferente valoración.

\section{Ámbito institucional}

Respecto del ámbito institucional, las licenciaturas que más valoran a la Universidad La Salle Pachuca fueron Enfermería (4.5), Ingeniería Cibernética (4.2) e Ingeniería Mecatrónica (4). Se considera que es un hallazgo digno de mencionar que aparte de Enfermería los alumnos de la Escuela de Ingeniería sean los que más valoran a la institución para la conformación de su CCU. Por otro lado, Ciencias de la Educación y TIN, valoran en un nivel medio la mencionada aportación (3.6 y 3.1 respectivamente).

Respecto a la variable la universidad fomenta el debate y el diálogo respetuoso la mitad de las licenciaturas valora en un nivel alto a la institución y la otra mitad en un nivel medio, entre las primeras están Enfermería (4.41) y Ciencias de la Educación (3.97), las cuales tienen diferencia significativa con Contaduría (3.47), Psicología (3.44) y TIN (2.50), que además la ubican en un nivel medio. Vuelve a llamar la atención que dos de las licenciaturas de la Facultad de Ciencias Humanas tengan diferente valoración. Se encuentra también el hecho de que las licenciaturas de la Facultad de Ciencias Administrativas valoren en un nivel medio esta variable, junto con Derecho y Diseño Gráfico.

El fomento a la crítica constructiva y autocrítica, es una variable en que la media aritmética global se encuentra en un nivel medio (3.62) y, en particular, las menores valoraciones se dan en Derecho y las licenciaturas de la Facultad de Ciencias Administrativas, con diferencias significativa con Enfermería y las licenciaturas de la Escuela de Ingeniería, donde parece haber un ambiente académico que favorece este proceso.

En relación con la libertad de pensamiento y la capacidad de expresión de demandas, de nueva cuenta Enfermería y las ingenierías valoraron más alto la aportación institucional y, junto con Arquitectura, tienen una diferencia significativa con las licenciaturas de la Facultad de Ciencias Administrativas y Ciencias de la Educación, las cuales obtuvieron los más bajos valores, ubicados además en un nivel medio. En la variable respeto por la legalidad, las puntuaciones más altas se 
obtuvieron en Enfermería, Ingeniería Cibernética y Ciencias de la Comunicación, con diferencia significativa en las puntuaciones de TIN y Ciencias de la Educación, que vuelven a ubicar este aspecto en un nivel medio.

La variable referida al fomento de cultura democrática en prácticas cotidianas sigue siendo valorada en primer término por Enfermería seguida de Ingeniería Mecatrónica, por otro lado, en un nivel medio la ubican las licenciaturas de la Facultad de Ciencias Administrativas, Ciencias de la Educación, Arquitectura y Psicología.

En cuanto a la realización de actos de solidaridad, sólo TIN valoró en un nivel medio y en Enfermería y Diseño Gráfico se obtuvieron las más altas puntuaciones. Cabe mencionar que la moda (opinión mayoritaria) fue cinco y esta variable fue una de las que obtuvieron mayor puntuación aun considerando los tres ámbitos. Por el contrario, la variable coherencia entre su ideario y sus prácticas cotidianas, fue de las que menor puntuación obtuvieron, ubicándose el promedio de todas las licenciaturas en un nivel medio. Enfermería e ingeniería Cibernética asignaron las valoraciones más altas con diferencia significativa con Derecho, TIN, Diseño Gráfico, Administración y Ciencias de la Educación.

En relación con la variable respeto de los derechos humanos de personas con diferentes maneras de pensar y/o vivir ninguna licenciatura otorgó una puntuación de nivel medio y la valoración mayoritaria fue la máxima, es decir, se obtuvo una moda de cinco, aunque Ciencias de la Educación vuelve a ser la licenciatura que menos valor otorgó. También la moda fue cinco en la variable rechazo a la discriminación y al racismo, aunque Psicología y Ciencias de la Educación la valoraron en un nivel medio con diferencia significativa con Ciencias de la Comunicación e Ingeniería Cibernética que fueron donde se obtuvieron los más altos promedios.

Para la capacidad para ejercer tus derechos ciudadanos excepto TIN, todas las licenciaturas la valoraron en un nivel alto y en cuanto a la justicia ante actos arbitrarios de autoridades y docentes tanto Enfermería, como TIN e Ingeniería Mecatrónica obtuvieron las valoraciones más altas con diferencia significativa con Administración y Ciencias de la Educación que valoraron esta variable en un nivel medio.

\section{Ámbito extraescolar}

En la valoración sobre su ámbito extraescolar, TIN, con sus dos alumnos, fue quien más alta puntuación obtuvo, sigue enfermería con su único semestre en ese momento. En tercer y cuarto lugar con promedios igual o mayor de cuatro, Derecho y Ciencias de la Educación. Por otro lado, con un nivel medio se encuentra Diseño Gráfico.

En relación con la variable soy capaz de reconsiderar o modificar puntos de vista con base en la obtención de información nueva, Contaduría y Enfermería se adjudican las mayores puntuaciones, TIN es la única en nivel medio. Respecto a 
soy capaz de involucrarme en la generación de proyectos de desarrollo con mi comunidad, sólo Contaduría se evalúa en un nivel medio, TIN y Ciencias de la Educación obtienen la puntuaciones más altas. En la variable conozco la situación sociopolítica de mi país, mi entidad federativa y mi municipio, TIN, Derecho y Contaduría obtienen las puntuaciones más altas con diferencia significativa con Enfermería y Diseño Gráfico, que además se ubican en un nivel medio junto con Ingeniería Cibernética.

Muy relacionada con la anterior variable es Conozco el nombre del gobernador, senador, diputado y presidente municipal que me representa, repiten primer y segundo lugar TIN y Derecho, con diferencia significativa con Ciencias de la Educación, Administración Ingeniería Mecatrónica, Diseño Gráfico e Ingeniería Cibernética. También Psicología se ubica en un nivel medio aunque sin diferencia significativa, es decir es una variable que la mitad de licenciaturas están en un nivel medio. Todavía más adhesión a un nivel medio esta la variable conozco acerca de los grupos y demandas sociales y políticas de la comunidad a la que pertenezco, ya que sólo TIN, Enfermería y Derecho se ubican en alto nivel, aunque con diferencia significativa con Ciencias de la Comunicación, Administración, Diseño Gráfico e Ingeniería Cibernética.

Caso contrario a lo anterior se encuentran las variables soy capaz de convivir con grupos humanos marginados y vulnerables, y soy capaz de vivir experiencias de socialización con grupos e individuos con diferentes puntos de vista o maneras de vivir al mío, en las que la opinión mayoritaria (moda) es la de más valor (cinco) y todas las licenciaturas se valoran en nivel alto.

En cuanto a la variable mis redes sociales presenciales y virtuales me proporcionan información de los problemas sociales y políticos de mi país,estado y municipio, sólo las licenciaturas de Contaduría, Ingeniería Mecatrónica y Diseño Gráfico se ubican en un nivel medio, con diferencia significativa con TIN, Enfermería y Ciencias de la Educación quienes obtuvieron las puntuaciones más altas. Relacionada con la anterior la variable participo en las redes sociales a las que pertenezco para apoyar u oponerme a demandas de tipo social y político se valoró en un nivel medio por todas las licenciaturas, excepto TIN. Cabe mencionar, que aún con esta valoración, Derecho (3.56) tiene diferencia significativa con Diseño Gráfico (3.04) e Ingeniería Mecatrónica (3.03), licenciaturas que obtuvieron los más bajos valores.

Finalmente, respecto a la variable ejerzo mis derechos y obligaciones ciudadanas en todas las licenciaturas se valoraron en un nivel alto, aunque con diferencia significativa entre TIN y Derecho (puntuaciones más altas) y Ciencias de la comunicación y Diseño Gráfico.

\section{Comentarios por licenciatura}

A continuación se presentan algunos comentarios de las licenciaturas de acuerdo con las variables involucradas. Lo anterior se hará de tal manera que cada párrafo se refiera a algún programa académico y se comenten aspectos generales acerca de los tres ámbitos que, bajo esta propuesta, conforman la CCU, contrastando alguna de las variables de alto nivel, con los de medio nivel y que tengan diferencia estadística significativa.

Los alumnos de Administración se consideran capaces de convivir con 
grupos marginados y vulnerables, así como con personas con diferente punto de vista o manera de vivir. También se consideran capaces de reconsiderar o modificar puntos de vista al obtener nueva información y creen ejercer plenamente sus derechos y obligaciones ciudadanas. Por otro lado, tienen una limitada participación en sus redes sociales para apoyar o rechazar demandas de tipo social o político, y conocen poco las que se generan en su comunidad. Consideran que sus estudios de licenciatura han sido muy importantes para generar un proyecto de vida y de autorrealización, además de valorar el conocimiento más como un medio de desarrollo humano que como mercancía. Estudiar Administración también ha sido fundamental en el conocimiento de sus derechos y obligaciones ciudadanas. Empero, consideran que ha sido limitado el desarrollo para localizar y valorar la calidad de fuentes de información, relacionar ésta y establecer hipótesis. En relación con el ámbito institucional, La Salle Pachuca fomenta el respeto de los derechos humanos independientemente de la manera de pensar o vivir, asimismo fomenta el rechazo a la discriminación y al racismo. Sin embargo, perciben que la institución tiene una limitada coherencia entre su ideario y sus prácticas cotidianas, así como un limitado fomento de la crítica constructiva, autocrítica, debate, diálogo, libertad de pensamiento, capacidad de expresión de demandas y cultura democrática en sus prácticas cotidianas. Los estudiantes de Arquitectura se autovaloran capaces de reconsiderar o modificar puntos de vista con base en obtener nueva información, así como de vivir experiencias de socialización con personas y grupos vulnerables, marginados, y con diferentes puntos de vista o maneras de vivir. También consideran que ejercen sus derechos y obligaciones ciudadanas. Sin embargo piensan que es limitada su participación en las redes sociales para la participación social o política y poco conocen acerca de los grupos o demandas sociopolíticas de su comunidad. El hecho de estudiar Arquitectura les impulsa a tomar decisiones reflexionadas y tener independencia de juicio, así como generar un proyecto de vida y autorrealización, considerando el conocimiento como medio de desarrollo humano y no como mercancía. Por el contrario, la licenciatura les ha brindado un aporte limitado para obtener una alta comprensión lectora y un alto nivel de expresión escrita; así como para relacionar información y establecer hipótesis. Es limitado también el aporte para reflexionar acerca de los mensajes de los medios de comunicación. La Universidad La Salle, para el alumnado de Arquitectura, fomenta el respeto de los derechos humanos sin excepción de personas, aun cuando haya diferencias de pensamiento o maneras de vivir, pero es limitada su cultura democrática y su coherencia entre el ideario y las prácticas cotidianas; y piensan que también es limitado el fomento de la institución para la crítica constructiva y la autocrítica.

Respecto a los jóvenes que cursan la Licenciatura en Ciencias de la Comunicación, éstos son capaces de vivir experiencias de socialización con grupos e individuos con diferentes puntos de vista o maneras de vivir a la de ellos, así como con grupos humanos marginados y vulnerables. También son capaces de reconsiderar o modificar opiniones con base en información nueva. Por otro lado es limitada su participación en las redes sociales con fines sociales o políticos y conocen poco a los grupos con demandas de este tipo en su comunidad. Valoran que el estudiar Ciencias de la Comunicación los hace 
reflexionar acerca del mensaje textual y la intención de los mensajes de los medios de comunicación; también consideran el conocimiento como un medio de desarrollo humano más que como mercancía. Asimismo la licenciatura ha sido importante para tomar decisiones reflexionadas, tener independencia de juicio y obtener una alta comprensión lectora y nivel de expresión escrita. Sin embargo, poco les aporta para conocer derechos y obligaciones ciudadanas. Piensan que la Universidad La Salle rechaza la discriminación y el racismo, fomenta el respeto por la legalidad y realiza actos de solidaridad. Por el contrario perciben que es limitada la coherencia entre el ideario institucional y sus prácticas cotidianas.

Para quienes estudian Ciencias de la Educación a través del ámbito extraescolar son capaces de vivir experiencias de socialización con grupos y personas que opinan diferente a ellos, que padecen marginación o son vulnerables socialmente. También son capaces de reconsiderar o modificar puntos de vista a través de nueva información, de ejercer sus derechos y obligaciones ciudadanas y consideran estar informados de la problemática sociopolítica del país, de la entidad federativa y municipio que les corresponde mediante sus redes sociales reales y virtuales. Sin embargo no participan en éstas para apoyar u oponerse a demandas políticas o sociales pues, además, tienen un conocimiento limitado de ellas y de los grupos que las promueven en su comunidad, así como de los políticos que los representan. Sus estudios de licenciatura han fomentado en ellos la generación de un proyecto de vida y autorrealización, consideran el conocimiento no como una mercancía, sino como medio de desarrollo humano, toman decisiones reflexionadas, tienen independencia de juicio y conocen derechos y obligaciones ciudadanas. Ninguna variable curricular la ubican en un nivel medio.

En cuanto a La Salle Pachuca, ésta realiza actos de solidaridad, pero consideran limitado el fomento de la justicia ante actos arbitrarios de autoridades y docentes, libertad de pensamiento y capacidad de expresión de demandas, coherencia entre su ideario y sus prácticas cotidianas, el rechazo a la discriminación y al racismo, y el respeto por la legalidad.

La percepción del alumnado de Contaduría es que conocen la situación sociopolítica del país, del estado Hidalgo y del municipio en el que viven, pues sus redes sociales presenciales y virtuales les proporcionan información, conocen a sus representantes políticos, y ejercen sus derechos y obligaciones ciudadanas. Sin embargo, es limitada su capacidad para involucrarse en la generación de proyectos de desarrollo, y contrario a la tendencia presentada, no son tan capaces de vivir experiencias de socialización con los "otros". Estudiar Contaduría les ha hecho conocer derechos y obligaciones ciudadanas; localizar, valorar, relacionar información y establecer hipótesis. En un nivel medio consideran la aportación de su programa en aspectos como generar un proyecto de vida y de autorrealización, así como de dotar al aprendizaje de sentido de vida. También consideran limitado el fomento de considerar el conocimiento como un medio de desarrollo humano, más que como una mercancía y el respeto por la legalidad. Como institución, consideran que La Salle fomenta la justicia, tiene una cultura democrática en sus prácticas cotidianas y realiza actos de solidaridad, además promueve la crítica constructiva y la autocrítica. Por otro lado, perciben limitaciones en el fomento de la libertad de pensamiento y la expresión de demandas, en el rechazo a la discriminación y al racismo, y al debate y diálogo respetuoso. 
En relación con los alumnos de Derecho, éstos consideran ejercer plenamente sus derechos y obligaciones ciudadanas; además son capaces de convivir con grupos humanos marginados y vulnerables, así como de vivir experiencias de socialización con grupos e individuos con diferentes puntos de vista. Suponen conocer la situación sociopolítica del país, del estado de Hidalgo (o al que pertenezcan) y su municipio, y por supuesto saben de los políticos que los representan.

Por otro lado, valoran como limitada su participación en las redes sociales a las que pertenecen para apoyar $u$ oponerse a demandas de tipo social y político. Sus estudios de licenciatura les han hecho conocer sus derechos y obligaciones ciudadanas y en ese ámbito existe un respeto por la legalidad. Asimismo, estudiar Derecho fomenta la toma de decisiones reflexionadas, la independencia de juicio, y la generación de un proyecto de vida y de autorrealización. Además se fomenta la resolución de problemas en diferentes condiciones y contextos, y la obtención de una alta comprensión lectora y nivel de expresión escrita. Cabe señalar que ninguna de las variables asociadas al ámbito curricular (licenciatura) la ubicaron en un nivel medio. En el ámbito institucional, se fomenta el ejercicio de los derechos ciudadanos, el respeto a los derechos humanos y a la legalidad. Sin embargo, ubican en un nivel medio la crítica constructiva y la autocrítica, la coherencia entre el ideario y las prácticas cotidianas; el debate, el diálogo, la libertad de pensamiento y la capacidad de expresión de demandas.

Respecto a los estudiantes de Diseño Gráfico, se perciben capaces de reconsiderar o modificar puntos de vista al obtener nueva información, así como de vivir experiencias de socialización con grupos e individuos con diferentes puntos de vista, maneras de vivir, marginados o grupos vulnerables. Con diferente valoración (nivel medio) ubican la participación sociopolítica en redes sociales, el conocimiento de grupos y demandas de la comunidad de pertenencia, de los políticos que los representan y en general, del conocimiento de la situación política y social del país, de la entidad federativa y del municipio de pertenencia. Estudiar la licenciatura de Diseño Gráfico, ha representado para el alumnado, el reflexionar acerca del mensaje textual y cifrado de los medios de comunicación, así como el tomar decisiones reflexionadas y tener independencia de juicio; también el resolver problemas en diferentes condiciones, el respeto por la legalidad, y el localizar y valorar la utilidad y calidad de las fuentes de información. En otro nivel (medio) ubican el fomentar el conocimiento de los derechos y obligaciones ciudadanas y el obtener una alta comprensión lectora y nivel de expresión escrita. Respecto a la Universidad La Salle, valoran que ésta fomenta la realización de actos de solidaridad y el respeto por la legalidad.

Sin embargo valoran limitado el fomento de la justicia ante actos arbitrarios, la coherencia entre el ideario y las prácticas cotidianas, la libertad de pensamiento y/o capacidad de expresión de demandas, así como el debate y el diálogo respetuoso.

En el caso de Enfermería, se perciben capaces de socializar con grupos y personas diferentes a ellos, aun los marginados y vulnerables. También son 
capaces de reconsiderar o modificar puntos de vista con base en nueva información, y creen ejercer sus derechos y obligaciones ciudadanas. En cambio consideran limitada su participación sociopolítica en las redes sociales a las que pertenecen y su conocimiento sobre la situación política y social del país, la entidad federativa y el municipio a que pertenecen. Los principales aspectos que la licenciatura fomenta son el respeto por la legalidad, el generar un proyecto de vida y de autorrealización, de dotar al aprendizaje de sentido de vida, tomar decisiones reflexionadas y caracterizar el contenido de las diversas fuentes de información. No hay variables curriculares que ubiquen en un nivel medio. Respecto a La Salle Pachuca, consideran que fomenta la realización de actos de solidaridad, el respeto por la legalidad, por los derechos humanos, la justicia ante actos arbitrarios y el ejercicio de sus derechos ciudadanos. Contrario a otras licenciaturas, consideran que sí hay libertad de pensamiento, capacidad de expresión de demandas y coherencia entre el ideario y las prácticas cotidianas, entre otros aspectos. Tampoco ubican ninguna variable del ámbito institucional en el nivel medio.

Ingeniería Cibernética tiene un alumnado que se considera capaz de vivir experiencias de socialización con grupos e individuos con diferentes puntos de vista o maneras de vivir al de ellos, y también de reconsiderar o modificar opiniones con base en nueva información. Sin embargo, tienen un limitado conocimiento acerca de los grupos y demandas sociales y políticas de la comunidad a la que pertenecen, así como de la situación sociopolítica del país, del estado y el municipio de pertenencia, y de los políticos que los representan. También es limitada su participación en las redes sociales para apoyar u oponerse a demandas. La licenciatura que estudian fomenta en ellos la capacidad de resolver problemas en diferentes contextos 0 condiciones, consideran el conocimiento como un medio de desarrollo humano más que como una mercancía. Sin embargo no les aporta tanto el conocimiento de los derechos y obligaciones ciudadanas. La institución, según su valoración fomenta la libertad de pensamiento, la capacidad de expresión de demandas, el respeto a los derechos humanos y a la legalidad; el rechazo a la discriminación y al racismo, su capacidad de ejercer sus derechos ciudadanos y la realización de actos de solidaridad. Ninguna variable ubicada en el ámbito institucional la valoran en un nivel medio.

Los estudiantes de Ingeniería Mecatrónica también se consideran capaces de socializar con personas diferentes a ellos, aun los marginados y vulnerables; ejercer sus derechos y obligaciones ciudadanas, y son capaces de reconsiderar o modificar puntos de vista al obtener información nueva. En cambio, es limitada su participación política y acceso de información sociopolítica en las redes sociales que participan, también limitado es el conocimiento de los políticos que los representan. Los estudios de la mencionada licenciatura fomenta en los alumnos el tomar decisiones reflexionadas y tener independencia de juicio, así como generar un proyecto de vida y de autorrealización, y resolver problemas en diferentes condiciones o contextos. Por otra parte, consideran limitado el obtener una alta comprensión lectora y nivel de expresión escrita, así como el reflexionar acerca del mensaje textual y la intención de los mensajes en los medios de comunicación, además del conocimiento de los derechos y obligaciones ciudadanas. Por su parte, la institución fomenta en el alumnado de Ingeniería Mecatrónica la realización de actos de solidaridad, la capacidad para ejercer sus derechos ciudadanos, el respeto a los derechos humanos, y sus prácticas 
cotidianas conllevan una cultura democrática. No ubican en el nivel medio ninguna variable incluida en el ámbito institucional.

Los estudiantes de Psicología se perciben capaces de reconsiderar o modificar puntos de vista con base en información nueva, también de convivir con grupos humanos marginados y vulnerables, así como de ejercer sus derechos y obligaciones ciudadanas. Sin embargo se consideran limitados para participar políticamente en sus redes sociales, en el conocimiento de grupos y demandas sociales que atañen a su comunidad, así como de los políticos que los representan. Sus estudios de Psicología les fomenta el generar un proyecto de vida y de autorrealización, así como de dotar al aprendizaje de un sentido de vida, de tomar decisiones reflexionadas, independencia de juicio, considerar el conocimiento como un medio de desarrollo humano más que como mercancía y el respeto por la legalidad. No valoran ningún aspecto considerado en el ámbito curricular con un nivel medio. La Salle Pachuca, si bien ha fomentado la realización de actos de solidaridad, tiene limitaciones para fomentar el debate y el diálogo, la crítica constructiva y la autocrítica, la libertad de pensamiento, la capacidad de expresión de demandas, el rechazo a la discriminación y al racismo, así como la cultura democrática en sus prácticas cotidianas.

En TIN, dos alumnos no son suficientes para determinar diferencias significativas estadísticamente, aun cuando sus valoraciones van de 4.5 (valoración para ocho variables extraescolares) a 1.5, es decir, abarcan también el nivel bajo, en el cual ubican la libertad de pensamiento y la capacidad de expresión de demandas, así como la cultura democrática en las prácticas cotidianas, en lo que se refiere a la institución. Respecto a la licenciatura consideran de bajo nivel el localizar y valorar la utilidad y calidad de diversas fuentes de información, y cabe aclarar que en este ámbito ninguna de las variables están en el nivel alto.

\section{Una mirada global}

De acuerdo con los resultados de esta encuesta, la capacidad de vivir experiencias de socialización con grupos e individuos con diferentes puntos de vista o maneras de vivir, es la variable con más alta valoración de acuerdo con el alumnado de licenciatura, muy relacionada con la variable que aparece en cuarto lugar referida a la capacidad de convivir con grupos humanos marginados y vulnerables, por lo que se reafirma lo que se había reportado en el estudio sobre cultura política de estudiantes de licenciatura de La Salle Pachuca (Mejía, 2009: 82) donde se estableció que "se considera también que elmencionado alumnado tiene

una franca proclividad hacia el multiculturalismo. Es decir de respeto hacia el otro, que se distingue ya sea por su condición social, cultural, religiosa o de preferencia sexual".

El ámbito curricular también es importante, al menos en las variables que se refieren a tomar decisiones reflexionadas y tener independencia de juicio (segundo lugar); generar un proyecto de vida y de autorrealización, así como dotar al aprendizaje de sentido de vida; respeto por la legalidad y considerar el conocimiento como un medio de desarrollo humano más que como una mercancía. Es decir, el alumnado le reconoce al ámbito curricular características y valores propios de una formación reflexiva y humanística. Respecto al ámbito institucional las tres principales variables fueron realización de 
actos de solidaridad; capacidad para ejercer derechos ciudadanos y respeto de los derechos humanos de personas con diferentes maneras de pensar y/o vivir. Las valoraciones ubicadas en el nivel medio (con diferencia significativa respecto a las mencionadas) y las de más bajo valor en el ámbito extraescolar se refieren a conocer acerca de los grupos y demandas sociales y políticas de la comunidad a la que pertenezco y participación en las redes sociales a las que pertenezco para apoyar $u$ oponerme a demandas de tipo social y político. Estas variables son consecuencia de cierta participación de tipo social o político, de tal manera que si seleccionamos los casos de los estudiantes que manifestaron participar en alguna organización social y política (58 casos válidos que representan el 12 por ciento del total de encuestados), ambas variables se ubican en un nivel alto.

Otras variables ubicadas en el nivel medio corresponden al ámbito institucional y se refieren a la coherencia entre su ideario y sus prácticas cotidianas; justicia ante actos arbitrarios de autoridades y docentes; y la crítica constructiva y la autocrítica, se considera que sería necesario otro tipo de análisis para profundizar en estos aspectos. Baste decir que estas tres variables se valoran en un nivel alto para Enfermería y las dos licenciaturas de la Escuela de Ingeniería.

\section{Conclusiones}

Este esfuerzo, finalmente invita a revisar, de cualquier forma, la Cultura Cívica Universitaria en aquellas Instituciones de Educación Superior interesadas en un verdadero perfil profesional ligado con la formación ciudadana de alto nivel. Ya se ha aclarado que la educación superior por sí misma no va a determinar el rumbo de la democracia, pero se considera que la puede matizar en un sentido más crítico, reflexivo e informado. Si bien ninguna investigación académica pretende descubrir realidades absolutas, es importante tomar en cuenta la percepción y valoración del estudiantado, ya que, al menos en este caso, se identificaron variables en las que en dicha valoración existe un acuerdo, si bien no generalizado, si con una clara tendencia que invita a estudiar las causas de determinada percepción. Por otro lado un instrumento de investigación como el aplicado, tiene la posibilidad de ser replanteado respecto a las variables y usado en estudios comparativos, de acuerdo con los objetivos específicos establecidos, en los cuales se pueden tomar en cuenta otros enfoques como la perspectiva de género, tiempo de estancia en la institución, ingreso económico, promedio escolar, etc. Por supuesto se reconoce la provisionalidad de los resultados obtenidos y la necesidad de su contrastación con otras técnicas de investigación tanto cuantitativas como cualitativas.

Más allá de las, en general, altas valoraciones del estudiantado de Universidad La Salle Pachuca y la discusión sobre el tratamiento estadístico de dicha valoración, el hallazgo de ciertas tendencias invita a profundizar acerca de las variables involucradas en ellas mediante otros enfoques y técnicas de investigación. Cabe aclarar que se llama tendencias a las variables con determinados parámetros estadísticos como lo son la media aritmética (y su ubicación en determinado nivel), la moda y la diferencia significativa que se repiten en las diferentes licenciaturas, o al menos, en las que integran determinada escuela o facultad, así como por el ámbito de ubicación.

Si bien existe una transversalidad de variables, en el sentido de que pueden 
abarcar los tres ámbitos (cuestión que se manifiesta con mayor claridad en los llamados ámbito curricular e institucional), dependerá de la situación contextual y del interés propio del investigador, el ubicarla en determinado ámbito y excluir o añadir otras variables. Asimismo, el tratamiento estadístico y la interpretación, pueden tener sus especificidades en razón del criterio institucional o del investigador involucrado. En suma, el planteamiento metodológico puede ser útil para diagnosticar el proceso de formación ciudadana en determinada Institución de Educación Superior, a fin de ubicar causalidades y, en su caso, generar procesos de cambios curriculares y generación u orientación de actividades extracurriculares.

Por último, se considera que para desarrollar el potencial de formación ciudadana en la Educación superior se requieren de mediaciones pedagógicas flexibles, dinámicas y abiertas, así como de la vinculación de los contenidos educativos con los acontecimientos sociales y personales cotidianos.

Es importante también la incorporación de contenidos de naturaleza humanística, así como la orientación cognitiva y formación de criterio a través de vivencias cotidianas de los jóvenes. Asimismo es necesario desarrollar técnicas didácticas basadas en el debate y participar en experiencias cognitivas y metacognitivas con sentido axiológico. Por otro lado es necesaria la utilización de espacios universitarios para la ejecución de proyectos autónomos por parte del estudiantado, así como el fomento de actividades colectivas relacionadas con al arte, la cultura, el deporte y la recreación. Es indispensable la formación de sujetos que sean constructores de su propio proceso individual y social, la educación de la voluntad para actuar de determinado modo y la creación de redes de aprendizaje mediante medios electrónicos y tradicionales.

La Educación Superior debe ampliar la capacidad de reflexión y la cosmovisión del alumnado, coadyuvar a considerar la política no sólo en términos de poder, sino como razonamiento y actuación de acuerdo con el interés colectivo e individual. Debe potenciar los procesos de socialización y vinculación no sólo con entidades de gobierno o la empresa con objetivos económicos, sino para crear o participar en organizaciones de la sociedad civil aun considerando que éstas tienen diferentes ideologías y fines. Finalmente es necesario visualizar la formación ciudadana en la Educación Superior como un proceso que reclama una teoría y una práctica educativa, para lograr que las universidades sean una verdadera conciencia crítica de la sociedad.

\section{FUENTES DE CONSULTA}

- ALMOND, A. y VERBA, S. (1992). La cultura política. En: Diez textos básicos de ciencia política, Espana: Ariel, p 171-201.

- BOLIVAR, A. (2005). El lugar de la ética profesional en la formación universitaria. En Revista Mexicana de Investigación Educativa, Ene-Mar 2005, Vol. 10, Núm. 024, p 93-123.

- HIRSCH, A. (2005). Construcción de una escala de actitudes sobre ética profesional. En: Revista Electrónica de Investigación Educativa, 7 (1). Recuperado en febrero de 2010, de http://redie.uabc.mx/vol7no1/contenido-hirsch.html. 
- MEJÍA, H. (2009). Cultura política de estudiantes de licenciatura en la Universidad La Salle Pachuca. En: Xihmai, vol. IV, número 08, Universidad La Salle Pachuca, pp. 55-86

- TEDESCO, J. C. (2003). Los pilares de la educación del futuro. En: Debates de Educación (2003: Barcelona, ponencia en línea). Fundación Jaume Bofill, UOC. Recuperado en junio de 2010, de http://www.uoc.edu/dt/20367/index.html.

- $\quad$ (2007). Inclusión social, nuevos procesosde socialización y ciudadanía social en América Latina, en: Calderón, Fernando (Coord.) Ciudadanía y Desarrollo Humano, Argentina: PNUD y siglo veintiuno editores.

- VElAsco, M. (2001). Un acercamiento al método tipológico en sociología. En: Tarres, M. Luisa (Coord.) Observar, escuchar y comprender sobre la tradición cualitativa en la investigación social. México: M.A. Porrúa, Colmex y Flacso.

- WEBER, M.(1984). Economía y Sociedad. México: FCE 